\title{
PERFIL DE SAÚDE DE IDOSOS INSERIDOS EM UMA UNIVERSIDADE DA MELHOR IDADE EM JUAZEIRO DO NORTE-CE.
}

\author{
HEALTH PROFILE OF ELDERLY INSTITUTED IN A UNIVERSITY OF THE THIRD AGE JUAZEIRO DO \\ NORTE-CE
}

BOTTCHER ${ }^{1}$, Lara Belmudes; SILVA, Maria Gessilania Rodrigues; FÉLIX, Maria Misleidy da Silva; José SILVA, Willyam de Sousa; SOARES, Maria Valeska de Sousa; BEZERRA, Marcos Antônio Araújo.

Centro Universitário Dr. Leão Sampaio

Recebido: 20/12/2017; Aceito: 02/02/2018; Publicado: 22/03/2018

\section{RESUMO}

Com o passar dos anos, a população mundial vem crescendo ainda mais em número de idosos. O envelhecimento é um conjunto de consequências ou efeitos da passagem do tempo, onde ocorrem consequências em todos os sistemas fisiológicos principais. Torna-se importante avaliar o perfil de indivíduos idosos afim de compreender quem são, para planejamento de estratégias favorecendo o cuidado com os idosos. Esta pesquisa caracteriza-se como sendo de campo, quantitativa-descritiva. A seleção da amostra foi do tipo intencional por conveniência composta por 66 idosas matriculadas na Universidade da Melhor Idade em Juazeiro do Norte-CE. Para obtenção das respostas aplicou-se uma anamnese e avaliação antropométrica. Os resultados indicaram que 80,3\% das mulheres encontravam-se com o IMC acima do peso, 80,3\% apresentaram o valor de RCQ maior que 0,85 o que indica um elevado risco cardíaco, $50 \%$ apresentam dores nas costas, $53 \%$ dores nas articulações, $54,6 \%$ apresentaram problemas de sono no último mês, 31,8\% responderam não ter estresse, $47 \%$ estresse leve e $10,6 \%$ estresse moderado. Quando questionadas sobre a prática de atividade física regular 71,2\% das mulheres dizem serem praticantes. A partir dos dados coletados na presente pesquisa é possível identificar que as idosas matriculadas na Universidade da Melhor Idade, apesar de praticantes de atividade física regular, apresentam sobrepeso, altos valores para razão cintura quadril, alterações no sono e consideráveis níveis de estresse. Dentre as doenças crônicas mais prevalentes encontra-se a Hipertensão Arterial seguida de Diabetes Mellitus.

Palavras-Chave: Idoso. Envelhecimento. Perfil de Saúde.

\begin{abstract}
Over the years, the world's population has grown even more in the number of elderly. Aging is a set of consequences or effects of the passage of time considered, where consequences occur in all major physiological systems. It is important to evaluate the profile of the use of elderly people in order to understand the planning of structures favoring care with the elderly. This research is characterized as being field, quantitative-descriptive. The selection of the sample for the type of intentional by convenience comprised 66 elderly women enrolled at the University of the Best Age in Juazeiro do Norte-CE. To obtain the answers anamnesis and anthropometric evaluation were applied. The results indicated that $80.3 \%$ of the women were overweight BMI, $80.3 \%$ had a WHR value greater than 0.85 indicating a high cardiac risk, $50 \%$ had back pain, 53 $54.6 \%$ had sleep problems in the last month, $31.8 \%$ said they did not have stress, $47 \%$ had mild stress and $10.6 \%$ had moderate stress. When questioned about regular physical activity practice, $71.2 \%$ of women say they are practicing. From the data collected in the present research, it is possible to identify that the elderly women enrolled in the University of the Best Age, despite regular physical activity, are overweight, have high waist ratio values, sleep disorders and considerable levels of stress. Among the most prevalent chronic diseases are Arterial Hypertension followed by Diabetes Mellitus.
\end{abstract}

Keywords: Elderly. Aging. Healthprofile.

${ }^{1}$ Av. Leão Sampaio Km 3, Lagoa Seca, Juazeiro do Norte-CE, CEP 63040-005. Email: larabottcher@leaosampaio.edu.br; Telefone (88) 981367477. 


\section{INTRODUÇÃO}

O envelhecimento populacional é um fenômeno cada vez mais importante e irreversível na sociedade. Segundo a Organização Mundial de Saúde em 2050 existirá um total de aproximadamente 2 bilhões de pessoas com mais de 60 anos sendo que destes $80 \%$ estarão nos países em desenvolvimento (SUZMAN et al.,2015).

O envelhecimento é um conjunto de consequências ou efeitos da passagem do tempo em que ocorrem declínios em todos os sistemas fisiológicos. Envelhecer também envolve mudanças e perdas biológicas as quais alteram a rotina do indivíduo, além de mudanças nos papéis e posições sociais e nas suas atividades diárias (DE ALCÂNTARA et al., 2014).

Dentre as principais alterações biológicas encontram-se as ocorridas no sistema cardiovascular, sistema respiratório, músculo-esquelético, nervoso e alterações no ciclo circadiano. Isso afeta diretamente a funcionalidade do idoso interferindo nas atividades de vida diária, além de relacionar-se diretamente com a incidência de doenças crônicas não transmissíveis (SILVA et al., 2014).

De acordo com Menezes et. al. (2013) o envelhecimento ocasiona várias mudanças corporais, tais como, diminuição do peso corporal, da estatura, massa muscular e o aumento e redistribuição da gordura corporal. Tais mudanças afetam a saúde dos idosos. A diminuição da massa muscular e o aumento da gordura corporal limitam os aspectos funcionais e incapacidades. $\mathrm{O}$ aumento no Índice de Massa Corporal e aumento na gordura visceral podem predispor essa população para doenças crônicas degenerativas, já que são fatores de risco para doenças cardíacas, hipertensão arterial, dislipidemia e diabetes mellitus.

Gobbi (2012) afirma que devido ao processo de envelhecimento as pessoas idosas tendem a aumentar o risco de desenvolver várias doenças. Vão perdendo sua capacidade funcional, no que resulta a diminuição de sua autonomia afetando negativamente na sua qualidade de vida.

Considerando o fenômeno do envelhecimento populacional torna-se importante conhecer o perfil da população idosa considerando diversos fatores, entre eles, a prevalência de doenças crônicas, perfil antropométrico, estilo de vida, alterações na qualidade do sono e níveis de estresse. O objetivo do presente trabalho foi traçar um perfil de idosos participantes da Universidade da Melhor idade, avaliando seu estilo de vida, prevalência de doenças crônicas e fatores de risco, estresse e alterações na qualidade do sono.

\section{MATERIAIS E MÉTODOS}

A pesquisa é considerada do tipo observacional, transversal, descritiva de abordagem quantitativa e respeitou as orientações da resolução 466/12 (BRASIL, 2012) que trata de pesquisa envolvendo seres humanos (Parecer de aprovação com protocolo CAAE $\mathrm{n}^{\circ}$. 2.378.936, no Comitê de Ética em Pesquisa do Centro Universitário Dr. Leão Sampaio - CEP/UNILEÃO ).

Participaram desse estudo 66 mulheres, com idade média de 65,8 \pm 6,1 anos, que participavam do Programa Universidade da Melhor Idade promovido pelo Centro Universitário Dr. Leão Sampaio em Juazeiro do Norte-CE.

A Universidade da Melhor Idade do Centro Universitário Dr. Leão Sampaio é um programa matutino que visa a inclusão e valorização da pessoa idosa e de acesso gratuito. As atividades acontecem duas vezes por semana, assegurando vivências socioeducativas, assistências, culturais e de lazer.

Adotou-se como critério de inclusão do presente estudo ser mulher com idade igual ou superior a 60 anos e participante regular da Universidade da Melhor Idade, enquanto que os critérios de exclusão dizem respeito ao pedido de retirar-se da pesquisa no momento que julgar-se conveniente, independente do motivo, não transparecer aptidão cognitiva para expressar de forma autônoma os questionamentos realizados (julgamento subjetivo do pesquisador com auxílio dos profissionais envolvidos no programa investigado).

Para obtenção dos dados para a pesquisa, aplicou-se uma anamnese composta de 14 questões relacionadas a fatores risco, doenças presentes, estilo de vida, prática de atividade física, qualidade do sono e estresse. Na sequência foi realizada uma avaliação para obtenção de medidas antropométricas de peso, estatura e circunferência de cintura e quadril seguindo o protocolo sugerido por Gordon et al (1991).

Foi realizada análise descritiva básica dos dados apresentando o valor percentual através do programa estatístico Statistical Package for the Social Sciences (SPSS) 20.0.

\section{RESULTADOS E DISCUSSÃO}

Para melhor visualização, os resultados foram apresentados em tabela (Tabela 1).

Tabela 1: Características Gerais Das Mulheres Estudadas

\begin{tabular}{lcc}
\hline Variáveis Categóricas & $\mathbf{N}$ & \% \\
\hline Eutrófico $\left(\mathrm{IMC}>18,5 \mathrm{e}<25 \mathrm{~kg} / \mathrm{m}^{2}\right)$ & 10 & 16,9 \\
Sobrepeso (IMC $\left.>25 \mathrm{e}<30 \mathrm{~kg} / \mathrm{m}^{2}\right)$ & 35 & 59,3 \\
Obesidade (IMC $\left.>=30 \mathrm{~kg} / \mathrm{m}^{2}\right)$ & 14 & 23,7 \\
& & \\
Risco Cintura-Quadril - Elevado Risco $(>0,85)$ & 53 & 80,3 \\
Risco Cintura-Quadril - Baixo Risco $(<0,85)$ & 13 & 19,6 \\
& & 51,5 \\
Hipertensão & 34 & 18,1 \\
Diabetes Mellitus & 12 & 10,6 \\
Angina & 7 & 6,0 \\
Doenças Cardíacas & 4 & 3,0 \\
Doenças Reumáticas & 2 & 1,5 \\
Doença Congênita & 1 &
\end{tabular}




$\begin{array}{lcc}\text { AVC } & 2 & 3,0 \\ \text { Tabagismo } & 5 & 7,5 \\ \text { Uso de bebida alcoólica } & 7 & 10,6 \\ \text { Problema Cardíaco } & 4 & 6,0 \\ \text { Desmaio } & 5 & 7,5 \\ \text { Problemas ósseos } & 29 & 43,9 \\ & & \\ \text { Dor nas costas } & 33 & 50,0 \\ \text { Dor nas articulações } & 34 & 53,0\end{array}$

\begin{tabular}{|c|c|c|}
\hline Variáveis Numéricas & Média & Desvio Padrão \\
\hline Idade(anos) & 65,80 & 6,15 \\
\hline $\operatorname{IMC}\left(\mathrm{kg} / \mathrm{m}^{2}\right)$ & 28,40 & 4,77 \\
\hline RCQ - Relação cintura-quadril & 0,92 & 0,06 \\
\hline PROBLEMAS DE SONO & $\mathbf{N}$ & $\%$ \\
\hline Nunca & 30 & 45,5 \\
\hline Menos de 1 vez/semana & 5 & 7,6 \\
\hline 1 ou 2 vezes/semana & 14 & 21,2 \\
\hline 3 ou mais vezes/semana & 17 & 25,8 \\
\hline NÍVEL DE ESTRESSE & $\mathbf{N}$ & $\%$ \\
\hline Sem estresse & 21 & 31,8 \\
\hline Estresse leve & 31 & 47,0 \\
\hline Estresse Moderado & 7 & 10,6 \\
\hline Estresse Constante & 4 & 6,1 \\
\hline Estresse Elevado & 3 & 4,5 \\
\hline ATIVIDADE FÍSICA & $\mathbf{N}$ & $\%$ \\
\hline PRÁTICA de atividade física regular & 47 & 71,2 \\
\hline \multicolumn{3}{|l|}{ Duração da Atividade Física } \\
\hline 40-60 Minutos & 18 & 38,3 \\
\hline 61-80 Minutos & 7 & 14,9 \\
\hline 81-100 Minutos & 6 & 12,8 \\
\hline$>100$ Minutos & 16 & 34,0 \\
\hline \multicolumn{3}{|l|}{ Atividade física práticada } \\
\hline Corrida & 2 & 3,0 \\
\hline Caminhada & 25 & 37,9 \\
\hline Bike & 2 & 3,0 \\
\hline Aeróbica & 9 & 13,6 \\
\hline Hidro & 10 & 15,2 \\
\hline Academia & 4 & 6,1 \\
\hline Musculação & 1 & 1,5 \\
\hline Outros & 13 & 19,7 \\
\hline
\end{tabular}

Fonte: Dados da pesquisa, 2017.

Foram avaliadas 66 mulheres idosas e através da obtenção das medidas de altura e peso foi possível calcular o Índice de Massa Corporal - IMC - na perspectiva de avaliar se esses idosos estavam com baixo peso, peso adequado ou sobrepeso. Conforme a tabela 1 , das mulheres avaliadas $16,9 \%$ encontravam-se com o
IMC eutrófico estando $83,1 \%$ acima do peso $(59,3 \%$ sobrepeso e $23,7 \%$ obesidade). Os resultados são preocupantes visto que a obesidade está associada ao surgimento de diversas doenças crônico degenerativas e risco de morte. 
Uma pesquisa do Ministério da Saúde, Vigitel (2014), apresentou resultados importantes ao indicar que o excesso de peso já atinge cerca de $52,5 \%$ da população adulta no Brasil. A pesquisa afirma que são necessárias medidas e o desenvolvimento de políticas públicas para combate ao sobrepeso e obesidade.

Lima (2014), em sua pesquisa, avaliou o IMC de 62 idosos com predominância de mulheres com idades entre 60 a 83 anos e verificou que $32 \%$ dos idosos apresentaram sobrepeso, $19 \%$ obesidade grau I e 5\% grau II, concluindo assim, que a maioria dos idosos estavam acima do peso.

Menezes et al (2013), alertam para o risco da população idosa de possuir IMC elevado. Segundo sua pesquisa, idosos que apresentam o IMC elevado podem desenvolver doenças como o Diabetes Mellitus, Hipertensão Arterial e doenças cardiovasculares, aumentando assim o risco de morte.

Diversos estudos já indicaram o risco de possuir um IMC acima dos valores normais. Uma pesquisa realizada por Di Angelantonio e um grupo de colaboradores (2016), analisaram 239 estudos com mais de 10 milhões de pessoas e os resultados indicam que um IMC alto aumenta significativamente as taxas de mortalidade. $\mathrm{O}$ estudo indica que indivíduos com obesidade apresentam expectativa de vida reduzida drasticamente.

$\mathrm{Na}$ presente pesquisa, outra variável analisada foi o Risco Cintura Quadril (RCQ). Sabe-se que RCQ é um preditor para doenças cardiovasculares pois indica a predominância de deposição de gordura abdominal e quanto maior o valor de RCQ, maior a distribuição de gordura abdominal e risco de doenças como hipertensão, aterosclerose, dislipidemias e eventos cardíacos. Para mulheres os valores de referência indicam que essa razão cintura quadril deve manter-se menor que 0,85 . Das idosas da Universidade da Melhor Idade, 80,3\% apresentaram o valor de RCQ maior que 0,85 o que indica um elevado risco cardíaco (ver tabela 1).

A anamnese da presente pesquisa também informou a prevalência de doenças crônicas sendo a mais prevalente a Hipertensão Arterial com 51,5\%, seguida do Diabetes Mellitus em 18,1\%, e na sequência as Doenças Cardíacas (Congênita e Reumática) com 10,5\%. Os problemas ósseos tiveram alta prevalência sendo citado por $43,9 \%$ das mulheres.

Machado et al (2017), avaliando 52 idosos vinculados a um grupo de convivência dos Centros de Saúde da Família, em um município do nordeste brasileiro, verificaram a prevalência de doenças crônicas e os hábitos de vida. Os resultados indicaram que a hipertensão arterial foi a principal doença crônica atingindo cerca de $42 \%$ dos homens e $30 \%$ das mulheres. Os dados relacionados ao estilo de vida indicaram que $58 \%$ das mulheres e $17 \%$ dos homens apresentaram sobrepeso ou obesidade e que $65 \%$ das mulheres e $92 \%$ dos homens não praticavam exercício físico. $\mathrm{O}$ autor concluiu que os dados da pesquisa são desfavoráveis na perspectiva de saúde com significativa prevalência de doenças crônicas degenerativas, assim como a quantidade de indivíduos que indicavam não praticar exercício físico, o que é fator de risco para desenvolvimento de doenças relacionadas ao sistema cardiovascular.

Um estudo realizado por Focchesatto et al (2015) avaliou 70 idosos de ambos os sexos, com idade igual ou acima de 60 anos, moradores de uma cidade do interior do Rio Grande do Sul. Após responderem uma anamnese e realizarem uma avaliação antropométrica os resultados apontaram que $76,5 \%$ apresentavam circunferência da cintura acima dos valores indicados para homens e mulheres, $55,9 \%$ apresentaram excesso de peso, $64,3 \%$ apresentaram hipertensão arterial.

Scherer et al (2013) analisaram 283 idosos, ativos socialmente de uma cidade do interior do Rio Grande do Sul. O objetivo do estudo foi avaliar o estado nutricional e prevalência de doenças. Mais uma vez os resultados indicaram alta prevalência de sobrepeso (60,3\% dos homens e $62,5 \%$ das mulheres) e quando avaliados a circunferência da cintura verificou-se risco muito alto para surgimento de doenças cardiovasculares em $92.5 \%$ das mulheres e $98,5 \%$ dos homens.

Considerando os dados da tabela 1 , das idosas da Universidade da Melhor Idade, 50\% apresentam dores nas costas e 53\% dores nas articulações. Esses resultados foram superiores aos encontrados em uma metanálise, realizada por Leopoldino et al. (2016) com objetivo de estimar a prevalência de dores nas costas em idosos. A pesquisa revisou 13 estudos originais com um total de 28.448 participantes e indicaram a prevalência de lombalgia em $25 \%$, dos indivíduos, resultado inferior aos encontrados no presente estudo.

Fernandes Melo et al (2017) verificou a prevalência de doenças musculares e esqueléticas auto relatas por 934 idosos em Goiás-GO. Os resultados indicaram que $39,1 \%$ dos idosos relataram possuir doenças músculo-esqueléticas e 43,4\% apontaram sentir dores. Os autores concluíram elevada prevalência de doenças músculo-esqueléticas nos idosos avaliados.

Em relação aos problemas de sono referentes ao último mês, das mulheres participantes da Universidade da Melhor Idade da Unileão, 45,5\% responderam que não tiveram problemas relacionados ao sono, $21,2 \%$ apresentaram problemas 1 ou 2 vezes, 7,6\% menos de 1 vez e $25,8 \%$ responderam que tiveram problemas com o sono de 3 ou mais vezes. Os resultados indicam que da amostra avaliada $54,6 \%$ apresentaram problemas de sono no último mês (tabela 1 ).

Sabe-se que o sono é um fenômeno natural e que dormir bem é fundamental para manter uma saúde equilibrada, devido seu papel importante na homeostasia do organismo. Entretanto o envelhecimento pode acarretar alterações no sono e consequentemente acentuar as queixas relacionadas ao mesmo (SCULLIN, 2015). É importante reconhecer que os problemas de sono acarretam o desequilíbrio em diversos sistemas fisiológicos ocasionando o surgimento de transtornos mentais, diminuição da competência imunológica, prejuízo no desempenho físico e dificuldades adaptativas. Essas alterações por sua vez, aumentam a vulnerabilidade do organismo do idoso, expondo consequentemente sua vida a riscos, surgimentos de doenças e problemas sociais ao indivíduo.

Dos Santos-Orlandi et al. (2016) mostraram através de seus achados que uma noite de sono insuficiente pode aumentar a sonolência diurna e prejudicar o idoso em relação à capacidade de manter-se em vigília durante todo o dia. $\mathrm{O}$ autor afirma que os problemas de sono são fatores de risco para doenças cardiovasculares, estágio de pré-fragilidade, sintomas depressivos, disfunção cognitiva e incapacidade funcional, aumentando a probabilidade de os indivíduos tornarem-se frágeis.

Já Silva (2014) analisou, dentre outras variáveis, a qualidade subjetiva do sono de 253 idosas e 
observou que a maior parte dos idosos avaliados $(52,3 \%)$ apresentaram qualidade de sono em níveis regular, ruim e muito ruim.

Existe relação entre obesidade, envelhecimento e alterações nos padrões de sono. Silva et al. (2014) estudaram 288 pacientes que foram avaliados quanto ao peso e estatura para cálculo do IMC, além de avaliados por polissonografia para identificação de distúrbios do sono. A partir dos resultados verificou-se relação entre IMC e distúrbios do sono já que a presença de obesidade aumentava o risco de distúrbio em três vezes. Os autores concluíram que a fragmentação do sono em indivíduos com apneia obstrutiva está associada a prevalência de excesso de peso sendo indicada sua redução imediata para essas pessoas.

Patel et al. (2014) avaliaram 3053 homens e 2985 mulheres acima de 60 anos e encontraram uma associação consistente entre alterações nos padrões de sono, o cochilo diurno e a obesidade, independente da duração média do sono. Como conclusão os autores indicaram inteiração direta entre sono, homeostase do peso e metabolismo corporal.

$\mathrm{Na}$ tabela 1 é possível verificar os dados relacionados aos níveis de estresse da amostra pesquisada. Ao serem questionadas sobre o nível de estresse diário, 31,8\% responderam não ter estresse, $47 \%$ estresse leve, $10,6 \%$ estresse moderado, $6,1 \%$ estresse constante e $4,5 \%$ estresse elevado.

Souza-Talarico et al. (2014) em seu estudo avaliaram 260 idosos com idades acima de 60 anos e ponderou alguns sintomas do estresse como por exemplo: ansiedade, esquecimento, cansaço, tristeza profunda, entre outros. A partir dos resultados de seu estudo, percebe-se que os idosos mais comprometidos cognitivamente apresentavam maiores concentrações de hormônios relacionados ao estresse (cortisol), maiores resultados quando avaliados a percepção de estresse e como consequência era o grupo que apresentavam problemas de memória mais frequentes.

Menezes-Silva et al. (2016) analisaram as correlações entre ansiedade, cognição, depressão, estresse percebido entre outras variáveis. Entre os 37 idosos avaliados, $21,6 \%$ foram classificados com depressão leve/moderada, 45,9\% apresentavam ansiedade, 78,4\% tinham comprometimento cognitivo. Existiu também, correlações de forma negativa entre idade e alterações cognitivas; e de forma positiva entre estresse e ansiedade e depressão e estresse.

As alterações sociais, emocionais e físicas decorrentes do envelhecimento são variáveis importantes para a incidência do estresse, interferindo na funcionalidade e atividades da vida diária, influenciando o bem-estar, independência e autonomia. Tais eventos podem gerar um quadro depressivo, alterações na qualidade do sono, instabilidade comportamental $\mathrm{e}$ redução da qualidade de vida. Além disso, diversos outros fatores associados ao processo de envelhecimento podem contribuir para o aumento do estresse entre eles o abandono por parentes, dependência e instabilidade financeira, surgimento das doenças crônicas degenerativas, morte de pessoas próximas, entre outros (GARBACCIO et al., 2014).

A última variável analisada, no presente estudo foi a prática regular de atividade física. Quando questionadas sobre a prática de atividade física regular $71,2 \%$ das mulheres dizem serem praticantes. Destas $38,3 \%$ relatam que quando o fazem, a duração fica entre
40-60 minutos, $14,9 \%$ entre $61-80$ minutos, $12,8 \%$ entre 81 e 100 minutos e $34 \%$ acima de 100 minutos. Quanto ao tipo de atividade física praticada, $37,9 \%$ realizam caminhada, $15,1 \%$ Hidroginástica, $13,6 \%$ Aeróbia e $33,4 \%$ outras atividades.

Existe consenso entre a comunidade científica de que a prática regular de atividade física para a população idosa garante diversos benefícios para suas vidas. Pitanga et al. (2018) afirmaram que a atividade física regular assume papel fundamental na prevenção e controle das doenças crônicas não transmissíveis, já que vários estudos relatam uma associação inversa entre atividade física e surgimento de tais comorbidades.

Sabe-se que a população idosa não adepta ao exercício físico está mais vulnerável aos acidentes do dia a dia. Devido a quantidade elevada de quedas, os idosos submetem-se à internações e reabilitações, ficam mais dependentes e em muitos casos chegam a óbito.

Santos et al. analisaram 280 idosos vinculados a Unidade Básica de Saúde no município de Natal-RN referente a prevalência de quedas e fatores associados. Do público avaliado $53,6 \%$ já haviam caído e destes $27,8 \%$ caíram duas ou mais vezes. Os resultados demonstraram maior prevalência de queda em idosos com performance física comprometida. A prática regular de atividade física diminui o risco de quedas resultando também em menos lesões e menos mortes.

Além disso, está bem estabelecido entre as pesquisas que a prática de atividade física melhora a saúde global do idoso, aumentando sua capacidade funcional, autonomia, garantindo maior independência e segurança para realização das atividades cotidianas. Consequentemente diminui o risco de doenças crônicas e melhora a saúde mental e física afetando positivamente na qualidade de vida dos idosos.

\section{CONCLUSÃO}

A partir dos dados coletados na presente pesquisa é possível verificar que as características encontradas nas idosas não diferem da literatura. Foi identificado que as idosas matriculadas na Universidade da Melhor Idade, apesar de praticantes de atividade física regular, apresentam sobrepeso, altos valores de razão cintura quadril, alterações no sono, e consideráveis níveis de estresse. Dentre as doenças crônicas mais prevalentes encontra-se a Hipertensão Arterial seguida de Diabetes Mellitus. Tais achados as colocam vulneráveis a diversos comprometimentos relacionados a saúde, principalmente em sua autonomia e independência.

Torna-se necessário a criação de medidas e estratégias que promovam a saúde de idosos, principalmente na prevenção e tratamento de doenças crônicas, promoção de hábitos saudáveis como prática regular de atividade física, alimentação saudável, cuidados com a qualidade do sono e redução dos níveis de estresse.

Conhecer o perfil desses idosos, a prevalência de doenças e fatores de riscos à saúde, permite a compreensão de suas reais necessidades garantindo o planejamento das ações para o cuidado ao idoso. Entretanto vale ressaltar que a população estudada está engajada em um projeto e por isso diferem de idosos institucionalizados ou inativos socialmente.

É importante que estratégias de alcance aos idosos que estão dentro de casa ou institucionalizados sejam promovidas visto que possivelmente estes estejam 
com perfil relacionado a saúde em níveis mais preocupantes.

\section{REFERÊNCIAS}

BRASIL. Ministério da Saúde. Conselho Nacional de Saúde. Resolução n. 466, de 12 de dezembro de 2012. Aprova diretrizes e normas regulamentadoras de pesquisas envolvendo seres humanos. Brasília, Diário Oficial da União, 12 dez. 2012.

DE ALCÂNTARA, Áyla Ribeiro et al. Análise comparativa qualidade de vida entre idosas praticantes e não-praticantes de atividade física em TeresinaPiauí. Revista Eletrônica Gestão \& Saúde, v. 5, n. 5, p. 3004-3014, 2014.

DE MENEZES, Tarciana Nobre et al. Perfil antropométrico dos idosos residentes em Campina Grande-PB. Revista Brasileira de Geriatria e Gerontologia, v. 16, n. 1, p. 19-27, 2013.

DI ANGELANTONIO, Emanuele et al. Body-mass index and all-cause mortality: individual-participant-data metaanalysis of 239 prospective studies in four continents. The Lancet, v. 388, n. 10046, p. 776-786, 2016.

DOS SANTOS-ORLANDI, Ariene Angelini et al. Factors associated with duration of naps among community-dwelling elderly: data from the multicenter study fibra. Texto \& Contexto Enfermagem, v. 25, n. 1, p. 1-10, 2016.

FECHINE, Basílio Rommel Almeida; TROMPIERI, Nicolino. O processo de envelhecimento: as principais alterações que acontecem com o idoso com o passar dos anos. InterSciencePlace, v. 1, n. 20, 2015.

FERNANDES MELO, Anna Cássia et al. Prevalência de doenças musculoesqueléticas autorreferidas segundo variáveis demográficas e de saúde: estudo transversal de idosos de Goiânia/GO. Cadernos Saúde Coletiva, v. 25, n. 2, 2017.

FOCCHESATTO, Andréia; CAMBOIM ROCKETT, Fernanda; SCHWEIGERT PERRY, Ingrid D. Fatores de risco e proteção para o desenvolvimento de doenças crônicas em população idosa rural do Rio Grande do Sul. Revista Brasileira de Geriatria e Gerontologia, v. 18, n. 4, 2015.

GARBACCIO, Juliana Ladeira; DA SILVA, Alanna Gomes; BARBOSA, Morgana Michella. Avaliação do índice de estresse em idosos residentes em domicílio. Northeast Network Nursing Journal, v. 15, n. 2, 2014.

GOBBI, Sebastião. Atividade física para pessoas idosas e recomendações da Organização Mundial de Saúde de 1996. Revista Brasileira de Atividade Física \& Saúde, v. 2, n. 2, p. 41-49, 2012.

GREGÓRIO, Paloma B. et al. Apresentação clínica de pacientes obesos com diagnóstico polissonográfico de apnéia obstrutiva do sono. Arq Bras Endocrinol Metabol, p. 1064-1068, 2007.

Brasil. Ministério da Saúde. Secretaria de Vigilância em Saúde. Departamento de Doenças e Agravos não transmissíveis e Promoção da Saúde. Vigilância de Fatores de Risco e Proteção para Doenças Crônicas por Inquérito Telefônico, Vigitel 2014. Brasília: Ministério da Saúde 2015.

LEOPOLDINO, Amanda Aparecida Oliveira et al. Prevalência de lombalgia na população idosa brasileira: revisão sistemática com metanálise. Revista Brasileira de Reumatologia, v. 56, n. 3, p. 258-269, 2016.

LIMA, Pollyanna Viana; DUARTE, Stenio Fernando Pimentel. Prevalência de obesidade em idosos e sua relação com hipertensão e diabetes. Revista InterScientia, v. 1, n. 3, p. 80-92, 2016.

MACHADO, Wyarlenn Divino et al. Idosos com doenças crônicas não transmissíveis: um estudo em grupos de convivência. Revista Ciência \& Saberes-Facema, v. 3, n. 2, p. 445-451, 2017.

MENEZES-SILVA, Rafael et al. Inquérito epidemiológico em população idosa (parte II): saúde bucal, ansiedade, depressão, estresse e uso de medicamentos. Scientia Medica, v. 26, n. 1, 2016.

PATEL, Sanjay R. et al. The association between sleep patterns and obesity in older adults. International journal of obesity, v. 38, n. 9, p. 1159, 2014.

PITANGA, Francisco José Gondim et al. Atividade Física no Tempo Livre, porém não Atividade Física no Deslocamento, está Associada com Risco Cardiovascular em Participantes do ELSA-Brasil. Arq Bras Cardiol, v. 110, n. 1, p. 36-43, 2018.

SANTOS, Roberta Kelly Mendonça dos et al. Prevalência e fatores associados ao risco de quedas em idosos adscritos a uma Unidade Básica de Saúde do município de Natal, RN, Brasil. Ciência \& Saúde Coletiva, v. 20, p. 3753-3762, 2015.

SCHERER, Rosangela et al. Estado nutricional e prevalência de doenças crônicas em idosos de um município do interior do Rio Grande do Sul. Revista Brasileira de Geriatria e Gerontologia, v. 16, n. 4, p. 769-779, 2013.

SCULLIN, Michael K.; BLIWISE, Donald L. Sleep, cognition, and normal aging: integrating a half century of multidisciplinary research. Perspectives on Psychological Science, v. 10, n. 1, p. 97-137, 2015. 
SILVA, Henyse et al. Factors associated with obstructive sleep apnea severity: obesity and excessive daytime sleepiness. International Journal of Cardiovascular Sciences, v. 27, n. 2, p. 76-82, 2014.

SILVA, Isabella Dantas da. Relação entre estresse percebido e qualidade de vida em pessoas idosas. 2014.

SOUZA-TALARICO, Juliana N. et al. Cross-country differences in basal and stress-induced cortisol secretion in older adults. PloS one, v. 9, n. 8, p. e105968, 2014.

SUZMAN, Richard et al. Health in an ageing worldwhat do we know?. The Lancet, v. 385, n. 9967, p. 484486, 2015. 